\title{
Network Pharmacology-based Prediction of Mechanism of Shenzhuo Formula for Application to DN
}

\section{Xin-miao Wang}

Department of Endocrinology, Guang'anmen Hospital, China Academy of Chinese Medical Sciences, Beijing, 100053, China

\section{Lin Han}

Department of Endocrinology, Guang'anmen Hospital, China Academy of Chinese Medical Sciences, Beijing, 100053, China

\section{Li-li Zhang}

Department of Endocrinology, Guang'anmen Hospital, China Academy of Chinese Medical Sciences, Beijing, 100053, China

\section{Sha Di}

Department of Endocrinology, Guang'anmen Hospital, China Academy of Chinese Medical Sciences, Beijing, 100053, China

\section{Xiu-xiu Wei}

Department of Endocrinology, Guang'anmen Hospital, China Academy of Chinese Medical Sciences, Beijing, 100053, China

\section{Hao-ran Wu}

Department of Endocrinology, Guang'anmen Hospital, China Academy of Chinese Medical Sciences, Beijing, 100053, China

\section{Hai-yu Zhang}

Department of Endocrinology, Guang'anmen Hospital, China Academy of Chinese Medical Sciences, Beijing, 100053, China

\section{Hao-yu Yang}

Department of Endocrinology, Guang'anmen Hospital, China Academy of Chinese Medical Sciences, Beijing, 100053, China

\section{Lin-hua Zhao}

Laboratory of Molecular and Biology, Guang'anmen Hospital, China Academy of Chinese Medical Sciences, Beijing, 100053, China

\section{Xiaolin Tong ( $\square$ tongxiaolin66@126.com )}

Department of Endocrinology, Guang'anmen Hospital, China Academy of Chinese Medical Sciences, Beijing, 100053, China 
Research

Keywords: Network pharmacology, Chinese medicine, Shenzhuo formula, Diabetic nephropathy, Mechanism

Posted Date: August 6th, 2020

DOI: https://doi.org/10.21203/rs.3.rs-53219/v1

License: (c) (i) This work is licensed under a Creative Commons Attribution 4.0 International License.

Read Full License 
Network Pharmacology-Based Prediction of Mechanism of Shenzhuo Formula for Application to DN

WANG Xin-miao a,1, HAN Lin ${ }^{\text {a, }}$, ZHANG Li-li a , DI Sha a, WEI Xiu-xiu a,b, WU

Hao-ran a,b, ZHANG Hai-yu ${ }^{\text {a,b }}$, YANG Hao-yu a,b ${ }^{\text {a, }}$ ZHAO Lin-hua ${ }^{\text {c,* }}$, TONG Xiao-lin $\mathrm{a},{ }^{* *}$

${ }^{a}$ Department of Endocrinology, Guang'anmen Hospital, China Academy of Chinese Medical Sciences, Beijing, 100053, China

${ }^{b}$ Graduate College, Beijing University of Traditional Chinese Medicine, Beijing, 100029, China

${ }^{c}$ Laboratory of Molecular and Biology, Guang'anmen Hospital, China Academy of Chinese Medical Sciences, Beijing, 100053, China

* Corresponding author.

** Corresponding author.

E-mail addresses: tongxiaolin66@126.com (X.-L. Tong), melonzhao@163.com (L.-H. Zhao).

${ }^{1}$ These authors have contributed equally to this work. 


\begin{abstract}
Background: Shenzhuo formula is a traditional Chinese medicine (TCM) prescription which has significant therapeutic effects on diabetic nephropathy (DN). However, its mechanism remains unknown. Therefore, this study aimed to explore the underlying anti-DN mechanism of shenzhuo formula.
\end{abstract}

Methods: The active ingredients and targets of shenzhuo formula were obtained by searching TCMSP, TCMID, SwissTargetPrediction and HIT. The DN target was identified from TTD, DrugBank and DisGeNet. The potential targets were obtained and PPI network were built after mapping the disease and drug targets. The key targets were screened out by network topology and the "drugs - DN - key targets" network was constructed by Cytoscape. GO analysis and KEGG pathway enrichment analysis were performed using DAVID, and the results were visualized using the Omicshare Tools. Results: We obtained 182 potential targets and 30 key targets. Ulteriorly, “drugs - DN key targets" network were constructed, and results showed that nodes like M51, M21, M5, M71, M28, EGFR, MMP9, MAPK8, PIK3CA and STAT3 had a higher degree. $\mathrm{GO}$ analysis results mainly involved in positive regulation of transcription from RNA polymerase II promoter, inflammatory response, lipopolysaccharide-mediated signaling pathway and other biological processes. The results of KEGG showed that DN-related pathways like TNF signaling pathway, PI3K-Akt signaling pathway were at the top of the list.

Conclusion: This article reveals the possible mechanism of shenzhuo formula in the treatment of DN through network pharmacology research, and lays a foundation for further studies.

\title{
Keywords:
}

Network pharmacology, Chinese medicine, Shenzhuo formula, Diabetic nephropathy, Mechanism 


\section{Introduction}

Diabetic nephropathy (DN) is one of the most common chronic microvascular complications of diabetes. It may be caused and shaped by the interaction of many factors such as endoplasmic reticulum dysfunction, high sugar-mediated generation of terminal advanced glycation endproducts (AGE), increased activation of the renin angiotensin aldosterone system, increased generation of reactive oxygen species (ROS), and activation of extracellular matrix (ECM) and protein kinase $\mathrm{C}[1-2]$. It is reported that the incidence of DN is about $40 \%$ in the diabetic population[3]. Furthermore, with the increasing incidence of diabetes, the incidence of DN is increasing yearly[4]. Therefore, it is important to intensify studies of the pathogenesis of DN and the search for effective intervention targets.

Shenzhuo formula as a traditional Chinese medicine (TCM) prescription has certain advantages in the treatment of DN[5]. It is created by Tong Xiaolin, an academician at the Chinese Academy of Sciences, and his research team. This formula was based on the pathogenesis of qi deficiency blood stasis, and the classic prescription of Didang decoction. Years of clinical practice have shown the effectiveness of shenzhuo formula where it can increase the glomerular filtration rate, reduce 24-hour urinary protein and kidney damage, and reverse kidney disease when used early[5-6]. However, due to the diversity of TCM compounds and complexity of in vivo processes, the systematic mechanism research of shenzhuo formula has been hindered .

Recently, network pharmacology aiming to predict pharmacological mechanisms has been developed rapidly with the use of multiomics, high-throughput screening, network visualization and analysis, or other techniques. It intuitively reveals the network structure of drug action[7], providing possibilities for exploring the mechanism of action of TCM compounds. Therefore, we planned to adopt network pharmacology 
method to preliminarily explore the mechanism of shenzhuo formula in preventing DN.

\section{Methods}

\subsection{Research tools}

The Chinese Traditional Medicine System Pharmacological Database Analysis Platform (TCMSP, http://lsp.nwu.edu.cn/tcmsp.php)[8], Traditional Chinese Medicine Integrated Database (TCMID, http://www.megabionet.org/tcmid/)[9], SwissTargetPrediction (http://www.swisstargetprediction.ch/)[10] and HIT (http:lifecenter.biosino.org/hit/)[11] were used to access to shenzhuo formula ingredients, targets. (2) The Therapeutic Target Database (TTD, http://bidd.nus.edu.sg/group/cjttd/)[12], DrugBank (https://www.drugbank.ca/)[13], and DisGeNet (http://www.disgenet.org/)[14] were used to get the target protein of DN. (3) The protein-protein interaction (PPI) network was obtained online using STRING (http://string-db.org)[15]. Compositional software Cytoscape 3.2.1 (http://www.cytoscape.org/)[16] was used to carry out network topology analysis and construct drug - DN - key target networks. The Database for Annotation, Visualization and Integrated Discovery (DAVID, http://david.ncifcrf.Gov)[17] was used for Gene Ontology (GO) and Kyoto Encyclopedia of Genes and Genomes (KEGG) analysis. The Omicshare Tools (https://www.Omicshare.com/) were used for visual analysis of GO and KEGG results.

\subsection{Collection of major chemical constituents}

This study relies on TCMSP, TCMID database and literatures mining to search for the chemical constituents of shenzhuo formula (Hedysarum Multijugum Maxim, Radix Salviae, Hirudo and Radix Rhei Et Rhizome).

\subsection{Screening of active compounds}

As we all know, TCM drugs reach into human body and take effect through absorption, distribution, metabolism, and excretion (ADME) processes. Among them, 
Oral bioavailability (OB) and drug similarity (DL), the key parameters of ADME components, were used as the screening criteria for active ingredients in this study. In this section, we operated it with TCMSP for which the ADME properties of each active molecule were collected in that data platform. And then the chemical constituents that meet the requirements of $\mathrm{OB} \geq 30 \%, \mathrm{DL} \geq 0.18$ were selected as potential active ingredients.

\subsection{Prediction of targets}

The SwissTargetPrediction and HIT web tools were used to collect the drug targets. In addition, TTD, DrugBank and DisGeNet databases were used to search for DN targets by entering the key words of diabetic kidney disease and diabetic nephropathy. Further, we matched the drug and DN targets to obtain the target database of "shenzhuo formula - DN - target".

\subsection{Network construction and analysis}

Based on the "shenzhuo formula - DN - target" database, the PPI network was obtained online using STRING. Furthermore, The PPI network topology analysis was carried out using Cytoscape 3.2.1 software and then key targets were obtained. To explore the sophisticated interactions between the active ingredients and their related targets at a system level, a "drugs - DN - key targets" network was constructed by Cytoscape3.2.1 software.

\subsection{GO and KEGG analysis}

The GO is widely used for gene function classification in the field of biology and mainly describes the molecular function (MF), biological processes (BP), and cellular components (CC) of genes. In this step, we use the DAVID tool for GO and KEGG pathway analysis. Then we used Omicshare Tools for visual display. 


\section{Results}

\subsection{Screening of candidate components in shenzhuo formula}

Through TCMSP, TCMID database, 87 species of compound Hedysarum Multijugum Maxim, 210 species of Radix Salviae, 35 species of Hirudo, 92 species of Radix Rhei Et Rhizome were obtained. Then the collected chemical constituents were calculated by $\mathrm{ADME}(\mathrm{OB} \geq 30 \%, \mathrm{DL} \geq 0.18)$ for further screening of active constituents (because the Hirudo could not be queried in TCMSP database, its ADME parameters could not obtained). After screening, one hundred and one active molecules were found, respectively 20 Hedysarum Multijugum Maxim, 65 in Radix Salviae, 16 in Radix Rhei Et Rhizome. In addition, through literature mining, another 4 active molecules were obtained, respectively 2 in Hedysarum Multijugum Maxim[18-19], 1 in Radix Salviae[20], 1 in Radix Rhei Et Rhizome[21].

\subsection{Target prediction}

Matching the targets of shenzhuo formula with 567 DN genes, a total of 182 potential targets of shenzhuo formula were obtained (Table 1).

Table 1

Potential anti-DN effects on gene targets of active component of shenzhuo formula (50 of 182 targets)

\begin{tabular}{|c|c|c|c|}
\hline Serial number & Target & $\begin{array}{l}\text { Common } \\
\text { name }\end{array}$ & Uniprot ID \\
\hline 1 & Aldose reductase & AKR1B1 & P15121 \\
\hline 2 & Acyl coenzyme A:cholesterol acyltransferase & CES1 & P23141 \\
\hline 3 & Signal transducer and activator of transcription 3 & STAT3 & P40763 \\
\hline 4 & Protein-tyrosine phosphatase $1 \mathrm{C}$ & PTPN6 & P29350 \\
\hline 5 & Vascular endothelial growth factor receptor 2 & KDR & P35968 \\
\hline 6 & Epidermal growth factor receptor erbB1 & EGFR & P00533 \\
\hline 7 & PI3-kinase p110-alpha subunit & PIK3CA & P42336 \\
\hline 8 & c-Jun N-terminal kinase 1 & MAPK8 & P45983 \\
\hline 9 & LXR-alpha & NR1H3 & Q13133 \\
\hline 10 & Estrogen receptor alpha & ESR1 & P03372 \\
\hline 11 & Testis-specific androgen-binding protein & SHBG & P04278 \\
\hline
\end{tabular}




\begin{tabular}{|c|c|c|c|}
\hline 12 & Cytochrome P450 2C19 & CYP2C19 & P33261 \\
\hline 13 & Protein-tyrosine phosphatase $1 \mathrm{~B}$ & PTPN1 & P18031 \\
\hline 14 & Butyrylcholinesterase & $\mathrm{BCHE}$ & P06276 \\
\hline 15 & Vitamin D receptor & VDR & P11473 \\
\hline 16 & Glucose-6-phosphate 1-dehydrogenase & G6PD & P11413 \\
\hline 17 & Peroxisome proliferator-activated receptor alpha & PPARA & Q07869 \\
\hline 18 & Peroxisome proliferator-activated receptor delta & PPARD & Q03181 \\
\hline 19 & Peroxisome proliferator-activated receptor gamma & PPARG & P37231 \\
\hline 20 & UDP-glucuronosyltransferase 2B7 & UGT2B7 & P16662 \\
\hline 21 & 11-beta-hydroxysteroid dehydrogenase 2 & HSD11B2 & P80365 \\
\hline 22 & NADPH oxidase 4 & NOX4 & Q9NPH5 \\
\hline 23 & Tyrosine-protein kinase SYK & SYK & P43405 \\
\hline 24 & Glycogen synthase kinase- 3 beta & GSK3B & P49841 \\
\hline 25 & Matrix metalloproteinase 9 & MMP9 & P14780 \\
\hline 26 & Matrix metalloproteinase 2 & MMP2 & P08253 \\
\hline 27 & Matrix metalloproteinase 12 & MMP12 & P39900 \\
\hline 28 & ATP-binding cassette sub-family G member 2 & $\mathrm{ABCG} 2$ & Q9UNQ0 \\
\hline 29 & P-glycoprotein 1 & $\mathrm{ABCB} 1$ & P08183 \\
\hline 30 & Arachidonate 12-lipoxygenase & ALOX12 & P18054 \\
\hline 31 & Cyclooxygenase- 2 & PTGS2 & P35354 \\
\hline 32 & Insulin-like growth factor I receptor & IGF1R & P08069 \\
\hline 33 & Myeloperoxidase & MPO & P05164 \\
\hline 34 & Matrix metalloproteinase 3 & MMP3 & P08254 \\
\hline 35 & Serine/threonine-protein kinase AKT & AKT1 & P31749 \\
\hline 36 & Beta-secretase 1 & BACE1 & P56817 \\
\hline 37 & Tyrosine-protein kinase receptor UFO & AXL & P30530 \\
\hline 38 & NUAK family SNF1-like kinase 1 & NUAK1 & O60285 \\
\hline 39 & Aldehyde reductase & AKR1A1 & P14550 \\
\hline 40 & Plasminogen & PLG & P00747 \\
\hline 41 & PI3-kinase p110-delta subunit & PIK3CD & O00329 \\
\hline 42 & PI3-kinase p110-gamma subunit & PIK3CG & P48736 \\
\hline 43 & Hematopoietic prostaglandin D synthase & HPGDS & O60760 \\
\hline 44 & Serine-protein kinase ATM & ATM & Q13315 \\
\hline 45 & Cytochrome P450 24A1 & CYP24A1 & Q07973 \\
\hline 46 & Mineralocorticoid receptor & $\mathrm{NR} 3 \mathrm{C} 2$ & P08235 \\
\hline 47 & Cannabinoid receptor 1 & CNR1 & P21554 \\
\hline 48 & Hepatocyte nuclear factor 4-alpha & HNF4A & P41235 \\
\hline 49 & C-C chemokine receptor type 1 & CCR1 & P32246 \\
\hline 50 & Histone-lysine N-methyltransferase EZH2 & EZH2 & Q15910 \\
\hline
\end{tabular}

Note: organism: Homo sapiens. Only 50 potential targets information was shown here, and the whole was in the appendix A. 


\subsection{Construction and analysis of network maps}

The PPI network of the 182 potential targets was obtained online using STRING (Fig. 1). Then we used Cytoscape 3.2.1 to obtained 30 key targets by network topology analysis with the inclusion criteria of "degree $\geq 2$ times of the median, closeness centrality $\geq$ median, betweenees centrality $\geq$ median" (Table 2 ). Next we constructed a “drugs - DN - key targets" network by Cytoscape 3.2.1 software (Fig. 2).

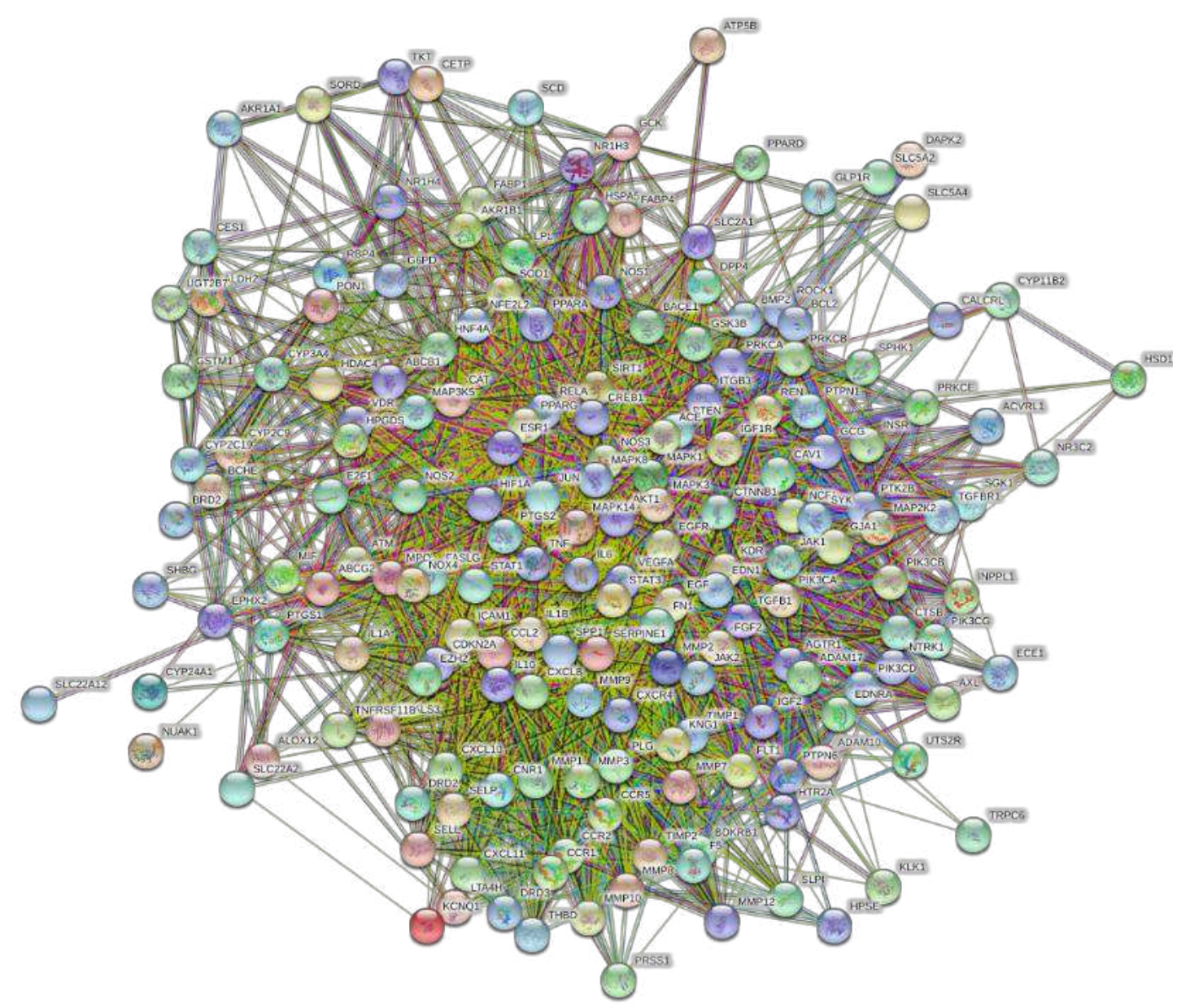

Fig. 1. PPI network of the 182 potential targets

Table 2

Thirty key targets obtained by network topology analysis

\begin{tabular}{|c|c|c|c|c|}
\hline serial number & node & Degree & Closeness centrality & Betweenees centrality \\
\hline
\end{tabular}




\begin{tabular}{|c|c|c|c|c|}
\hline 1 & PIK3CA & 40 & 0.49508197 & 0.09370214 \\
\hline 2 & STAT3 & 40 & 0.5 & 0.0863086 \\
\hline 3 & AKT1 & 35 & 0.49025974 & 0.15311921 \\
\hline 4 & KNG1 & 33 & 0.44023324 & 0.06128185 \\
\hline 5 & VEGFA & 33 & 0.491856668 & 0.06953442 \\
\hline 6 & JUN & 32 & 0.48089172 & 0.07229449 \\
\hline 7 & MAPK3 & 30 & 0.4617737 & 0.02240476 \\
\hline 8 & MAPK1 & 30 & 0.4689441 & 0.06714477 \\
\hline 9 & EGF & 27 & 0.4617737 & 0.0336672 \\
\hline 10 & EDN1 & 27 & 0.46604938 & 0.05180077 \\
\hline 11 & EGFR & 26 & 0.44023324 & 0.01794429 \\
\hline 12 & JAK1 & 26 & 0.44940476 & 0.02254905 \\
\hline 13 & IL6 & 26 & 0.45209581 & 0.02532622 \\
\hline 14 & CXCL8 & 25 & 0.43768116 & 0.03191743 \\
\hline 15 & RELA & 24 & 0.45757576 & 0.04035241 \\
\hline 16 & FN1 & 23 & 0.4351585 & 0.01464828 \\
\hline 17 & JAK2 & 23 & 0.44940476 & 0.01620852 \\
\hline 18 & CTNNB1 & 23 & 0.45481928 & 0.06488997 \\
\hline 19 & TNF & 22 & 0.44281525 & 0.0272631 \\
\hline 20 & TGFB1 & 21 & 0.44281525 & 0.03270724 \\
\hline 21 & MMP9 & 20 & 0.40921409 & 0.03200512 \\
\hline 22 & CXCR4 & 19 & 0.41032609 & 0.01402652 \\
\hline 23 & TIMP1 & 19 & 0.41712707 & 0.00798146 \\
\hline 24 & MAPK14 & 19 & 0.44411765 & 0.01628416 \\
\hline 25 & BDKRB1 & 18 & 0.3994709 & 0.00725308 \\
\hline 26 & PIK3CB & 18 & 0.40921409 & 0.00732155 \\
\hline 27 & MAPK8 & 18 & 0.42296919 & 0.03099697 \\
\hline 28 & ITGB3 & 18 & 0.42296919 & 0.01050834 \\
\hline 29 & CCR5 & 16 & 0.39841689 & 0.00592392 \\
\hline 30 & PLG & 16 & 0.402666667 & 0.02168017 \\
\hline
\end{tabular}




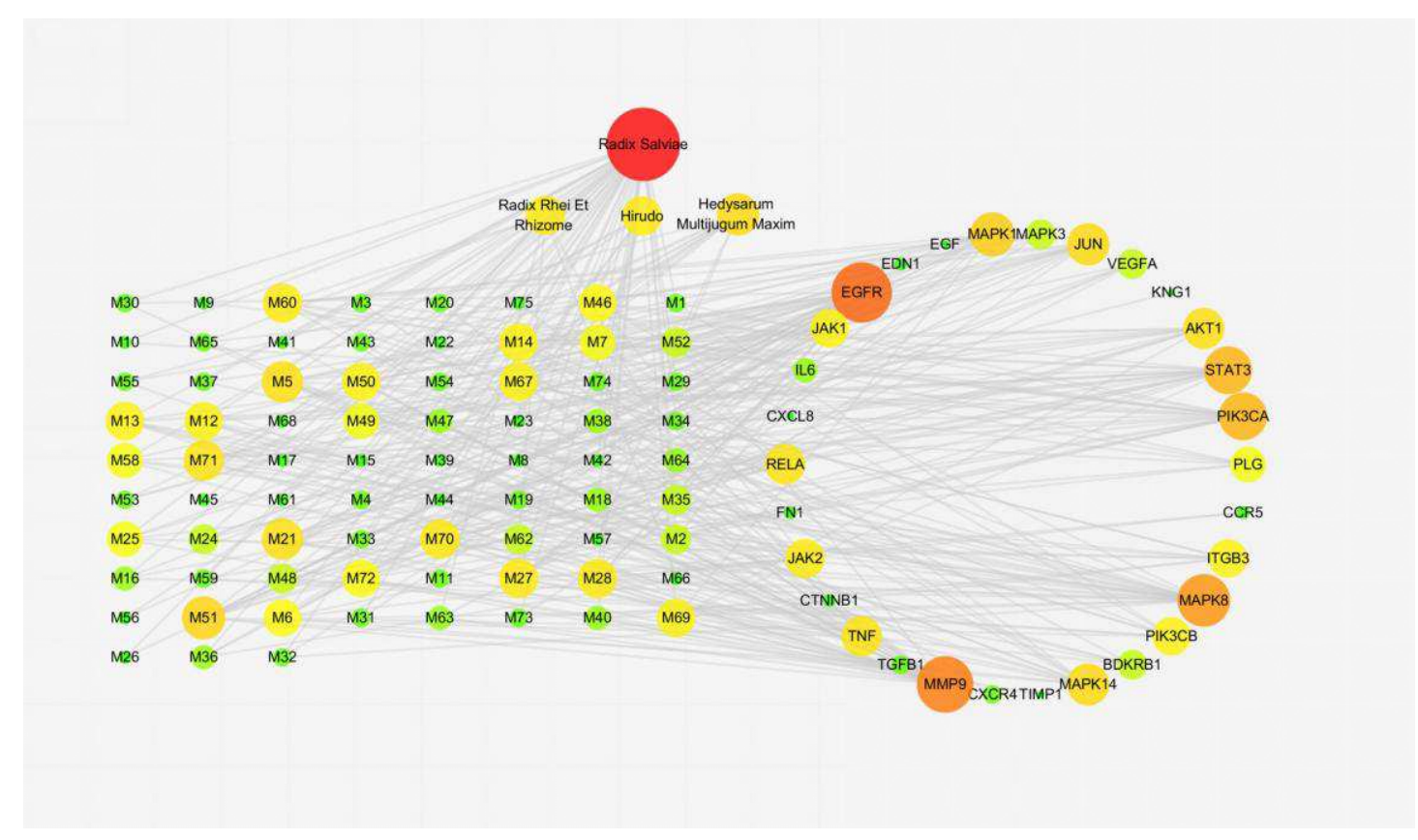

Fig. 2. "Drugs - DN - key targets" network. The nodes were visualized with degree. The larger and the redder the node, the higher the degree it was. M1-75 stand for the active ingredients which specific names were shown at appendix B.

\subsection{GO and KEGG analysis}

The DAVID tool was used to do the GO analysis. And the GO terms were constructed by the Omicshare Tools (Fig. 3). The GO analysis results showed that the targets were mainly involved in positive regulation of transcription from RNA polymerase II promoter, inflammatory response, lipopolysaccharide-mediated signaling pathway, positive regulation of peptidyl-serine phosphorylation and other biological processes. As the top $20 \mathrm{GO}$ enrichment items listed, suggesting that $\mathrm{DN}$ is relevent to scores of BP in body abnormalities, shenzhuo formula is likely to regulate these items and then play an anti-DN role. 


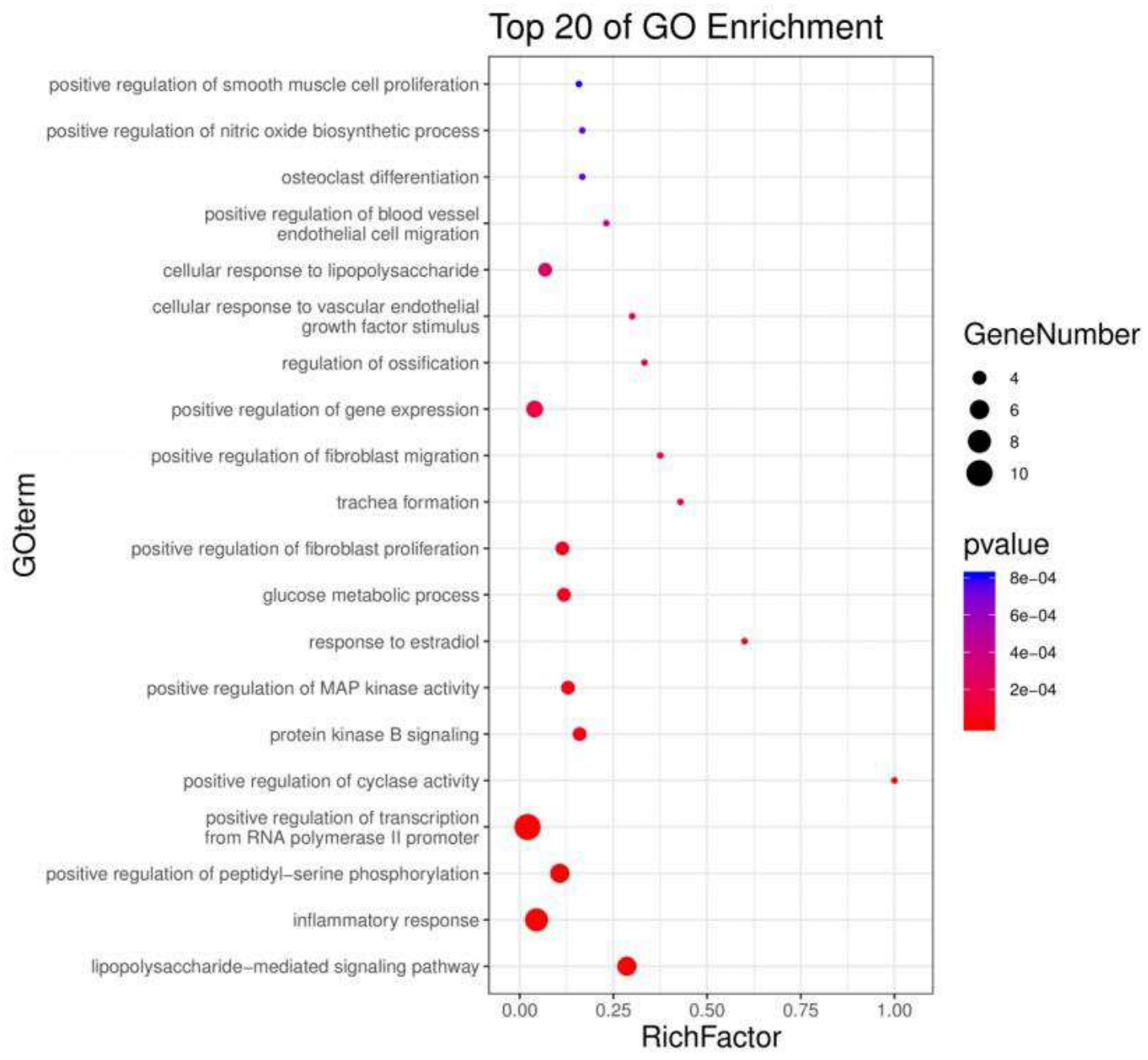

Fig. 3. Top 20 enrichments in GO analysis

The DAVID online tool was used to conduct the KEGG pathway enrichment analysis of the screened 30 key target proteins, and a total of 104 enrichment results were obtained. Fig. 4 is the top 20 enrichment analysis of KEGG pathway for predicting the anti-DN effect of shenzhuo formula. It showed that 20 targets of key targets were involved in the Pathways in cancer (20/30,66.7\%), 15 targets were involved in Hepatitis B (15/30, 50.0\%), 15 targets were involved in Influenza A (15/30, 50.0\%), 15 targets involved in Proteoglycans in cancer (15/30, 50.0\%), 13 targets involved in TNF signaling pathway $(13 / 30,43.3 \%)$ and 13 targets involved in PI3K-Akt signaling pathway $(13 / 30,43.3 \%)$. 


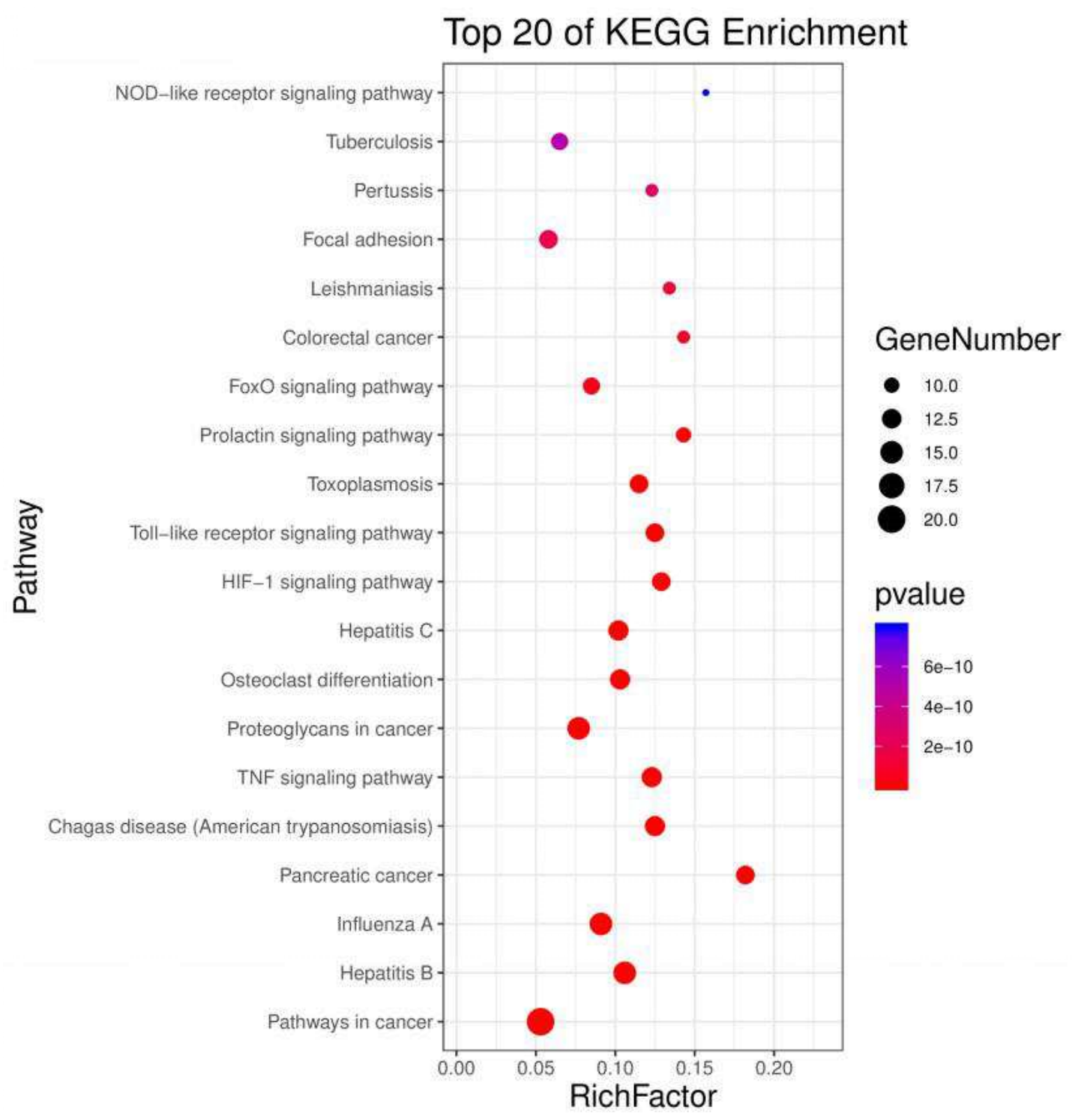

Fig. 4. Top 20 KEGG pathway enrichments

\section{Discussion}

Previous studies have suggested that shenzhuo formula has a good therapeutic effect on DN. However, the potential mechanisms of shenzhuo formula treating in DN have not been fully explained. In this study, we mainly applied network pharmacology method to explore it. To be more specific, 140 potential active compounds and their related 182 potential targets were obtained after pharmacokinetic screening and DN related target mapping, which will be contribute to the further research of this formula. Then, we constructed two networks, including the PPI of 182 potential targets network 
and drug - DN - key target network, and applied GO and KEGG enrichment analysis to explore the regulation of shenzhuo formula for treating DN.

Through the drug - DN - key target network, we can know that most ingredients are linked to no less than one target, which tells us that TCM ingredients have a property of multitargets. The result of the drug - DN - key target network analysis has suggested that different active compounds from different herbs can act on the same one target, which might demonstrate that shenzhuo formula has a synergistic effect during treating DN. In addition, the results of the drugs - DN - targets network topology analysis showed that there are 8 active ingredients which degrees were greater than 2 times of the average. Interestingly, 3 of these have been experimentally proven to have kidney protection effect. For example, quercetin liposomes has renal protective effects by reducing oxidative stress, attenuating AGE expression, and delaying the progression of $\mathrm{DN}[22]$. Luteolin attenuates DN mainly via suppression of inflammatory response and oxidative response[23]. Ursolic acid alleviated renal damage in type 2 diabetic $\mathrm{db} / \mathrm{db}$ mice by downregulating proteins in the angiotensin II type 1 receptor-associated protein / angiotensin II type 1 receptor signaling pathway to inhibit extracellular matrix accumulation, renal inflammation, fibrosis and oxidative stress[24]. These results accord closely with our predictions, which suggested that the ingredients with higher degree might play an important role in the treatment of DN. Meanwhile, we discovered five ingredients (M5, M27, M28, M60 and M70) are likely to have renal protection effect but haven't been verified up to now.

Moreover, the results of the drugs - DN - targets network topology analysis also showed that there are 5 targets which degrees were greater than 2 times of the average. Particularly, 3 of these have been experimentally proven to be in contact with DN. For instance, EGFR activation has a significant role in activating pathways that mediate podocyte injury and loss in diabetic nephropathy[25]. Down-regulated expression of 
MMP-9 can promote the process of DN[26]. STAT3 inhibition can hinder the development and progression of DN in diabetic patients[27].

As shown in the GO enrichment analysis results, the potential targets of shenzhuo formula acting on DN were mainly associated with various biological processes, such as lipopolysaccharide-mediated signaling pathway, inflammatory response, positive regulation of cyclase activity, protein kinase B signaling, positive regulation of MAP kinase activity, response to estradiol, which have a strongly direct correlation with the pathogenesis of DN[28-34].

Similarly, the results of KEGG enrichment analysis showed that shenzhuo formula takes an effect in the treatment of DN through multiple pathways. Through further research, we found that these pathways have been experimentally confirmed, such as TNF signaling pathway[35], HIF-1 signaling pathway[36], Toll-like receptor signaling pathway[37], FoxO signaling pathway[38], Focal adhesion[39], NOD-like receptor signaling pathway[40] and other ways to exert anti-DN potential. These results were also consistent with the result predicted by the network analysis. In addition, the KEGG enrichment analysis suggested that shenzhuo formula may have potential therapeutic effects on diseases such as cancer, hepatitis, influenza, leishmaniasis, pertussis and tuberculosis. As is reported that different diseases have common or similar pathological changes and can be treated with the same prescription[41], the above results suggest that shenzhuo formula concentrates more on the systematicness of the body when treating DN. in other words, shenzhuo formula possibly regulates the body to reach the balance state, then reaching the aim of treatment.

\section{Conclusion}

In conclusion, this study based on the network pharmacology, has preliminary explained the anti - DN mechanism of shenzhuo formula from the perspective of 
multi-active ingredients, multi-targets, multi-pathway. And it also provides reference for further research and might be benefit for shenzhuo formula's clinical application to some extent.

\title{
Supplementary Materials
}

Appendix A: Table. S1. A total of 182 potential targets.

Appendix B: Table. S2. The code information for active ingredients

\begin{abstract}
Abbreviations
TCM: traditional Chinese medicine; DN: diabetic nephropathy; AGE: advanced glycation endproducts; ROS: reactive oxygen species; ECM: extracellular matrix; TCMSP: the Chinese Traditional Medicine System Pharmacological Database Analysis Platform; TCMID: Traditional Chinese Medicine Integrated Database; TTD: Therapeutic Target Database; PPI: protein-protein interaction; DAVID: Database for Annotation, Visualization and Integrated Discovery; GO: Gene Ontology; KEGG: Kyoto Encyclopedia of Genes and Genomes; ADME: absorption, distribution, metabolism and excretion; OB: Oral bioavailability; DL: drug similarity; MF: molecular function; BP: biological processes; CC: cellular components
\end{abstract}

\section{Acknowledgements}

We thank Wen-jing He for her writing assistance.

\section{Patient consent for publication}

Not applicable.

\section{Authors' contributions}

XT and LZ designed the study; XW and LH wrote the paper; LZ, XW, HZ and HY performed the study and analyzed the data; SD and HW supervised the study and 
revised the manuscript. All authors read and approved the final manuscript.

\section{Funding}

This study was supported by the Fundamental Research Funds for the China Academy of Chinese Medical Sciences (No. ZZ0808004).

\section{Availability of data and materials}

The data used to support the results of this study can be obtained from the corresponding author upon reasonable request.

\section{Ethics approval and consent to participate}

Not applicable.

\section{Consent for publication}

Not applicable.

\section{Competing interest}

There is no conflict of interest declared.

\section{References:}

1. Cao AL, Wang L, Chen X, Wang YM, Guo HJ, Chu S, et al. Ursodeoxycholic acid and 4-phenylbutyrate prevent endoplasmic reticulum stress-induced podocyte apoptosis in diabetic nephropathy. LAB INVEST. 2016 2016-06-01;96(6):610-22.

2. Sun GD, Cui WP, Guo QY, Miao LN. Histone lysine methylation in diabetic nephropathy. J DIABETES RES. 2014 2014-01-20;2014:654148.

3. Alicic RZ, Rooney MT, Tuttle KR. Diabetic Kidney Disease: Challenges, Progress, and Possibilities. Clin J Am Soc Nephrol. 2017 2017-12-07;12(12):2032-45.

4. Magee C, Grieve DJ, Watson CJ, Brazil DP. Diabetic Nephropathy: a Tangled Web to Unweave. Cardiovasc Drugs Ther. 2017 2017-12-01;31(5-6):579-92.

5. Chen H, Guo J, Zhao X, He X, He Z, Zhao L, et al. Retrospective analysis of the overt proteinuria diabetic kidney disease in the treatment of modified Shenzhuo formula for 2 years. Medicine (Baltimore). 2017 2017-03-01;96(12):e6349.

6. Tian J, Zhao L, Zhou Q,et al. Retrospective analysis on modified Didang Tang for treating 
microalbuminuria of diabetic nephropathy. J Beijing Univ Tradit Chin Med (Clinical Medicine), 2012 19(6):7-10 .

7. Hopkins AL. Network pharmacology: the next paradigm in drug discovery. NAT CHEM BIOL. 2008 2008-11-01;4(11):682-90.

8. Ru J, Li P, Wang J, Zhou W, Li B, Huang C, et al. TCMSP: a database of systems pharmacology for drug discovery from herbal medicines. J Cheminform. 2014 2014-01-20;6:13.

9. Xue R, Fang Z, Zhang M, Yi Z, Wen C, Shi T. TCMID: Traditional Chinese Medicine integrative database for herb molecular mechanism analysis. NUCLEIC ACIDS RES. 2013 2013-01-01;41(Database issue):D1089-95.

10. Daina A, Michielin O, Zoete V. SwissTargetPrediction: updated data and new features for efficient prediction of protein targets of small molecules. NUCLEIC ACIDS RES. 2019 2019-07-02;47(W1):W357-64.

11. Ye H, Ye L, Kang H, Zhang D, Tao L, Tang K, et al. HIT: linking herbal active ingredients to targets. NUCLEIC ACIDS RES. 2011 2011-01-01;39(Database issue):D1055-9.

12. Li YH, Yu CY, Li XX, Zhang P, Tang J, Yang Q, et al. Therapeutic target database update 2018: enriched resource for facilitating bench-to-clinic research of targeted therapeutics. NUCLEIC ACIDS RES. 2018 2018-01-04;46(D1):D1121-7.

13. Wishart DS, Feunang YD, Guo AC, Lo EJ, Marcu A, Grant JR, et al. DrugBank 5.0: a major update to the DrugBank database for 2018. NUCLEIC ACIDS RES. 2018 2018-01-04;46(D1):D1074-82.

14. Pinero J, Bravo A, Queralt-Rosinach N, Gutierrez-Sacristan A, Deu-Pons J, Centeno E, et al. DisGeNET: a comprehensive platform integrating information on human disease-associated genes and variants. NUCLEIC ACIDS RES. 2017 2017-01-04;45(D1):D833-9.

15. Szklarczyk D, Gable AL, Lyon D, Junge A, Wyder S, Huerta-Cepas J, et al. STRING v11: protein-protein association networks with increased coverage, supporting functional discovery in genome-wide experimental datasets. NUCLEIC ACIDS RES. 2019 2019-01-08;47(D1):D607-13.

16. Shannon P, Markiel A, Ozier O, Baliga NS, Wang JT, Ramage D, et al. Cytoscape: a software environment for integrated models of biomolecular interaction networks. GENOME RES. 2003 2003-11-01;13(11):2498-504.

17. Huang DW, Sherman BT, Lempicki RA. Systematic and integrative analysis of large gene lists using DAVID bioinformatics resources. NAT PROTOC. 2009 2009-01-20;4(1):44-57.

18. Zhou L, Liu Z, Wang Z, Yu S, Long T, Zhou X, et al. Astragalus polysaccharides exerts immunomodulatory effects via TLR4-mediated MyD88-dependent signaling pathway in vitro and in vivo. Sci Rep. 2017 2017-03-17;7:44822.

19. Li L, Hou X, Xu R, Liu C, Tu M. Research review on the pharmacological effects of astragaloside IV. Fundam Clin Pharmacol. 2017 2017-02-01;31(1):17-36.

20. Bao XY, Zheng Q, Tong Q, Zhu PC, Zhuang Z, Zheng GQ, et al. Danshensu for Myocardial Ischemic Injury: Preclinical Evidence and Novel Methodology of Quality Assessment Tool. FRONT PHARMACOL. 2018 2018-01-20;9:1445.

21. Dong X, Fu J, Yin X, Cao S, Li X, Lin L, et al. Emodin: A Review of its Pharmacology, Toxicity and Pharmacokinetics. PHYTOTHER RES. 2016 2016-08-01;30(8):1207-18.

22. Tang L, Li K, Zhang $\mathrm{Y}, \mathrm{Li} \mathrm{H}, \mathrm{Li} \mathrm{A}, \mathrm{Xu} \mathrm{Y}$, et al. Quercetin liposomes ameliorate streptozotocin-induced diabetic nephropathy in diabetic rats. Sci Rep. 2020 2020-02-12;10(1):2440.

23. Zhang M, He L, Liu J, Zhou L. Luteolin Attenuates Diabetic Nephropathy through Suppressing 
Inflammatory Response and Oxidative Stress by Inhibiting STAT3 Pathway. Exp Clin Endocrinol Diabetes. 2020 2020-01-02.

24. Ma TK, Xu L, Lu LX, Cao X, Li X, Li LL, et al. Ursolic Acid Treatment Alleviates Diabetic Kidney Injury By Regulating The ARAP1/AT1R Signaling Pathway. Diabetes Metab Syndr Obes. 2019 2019-01-20;12:2597-608.

25. Chen J, Chen JK, Harris RC. EGF receptor deletion in podocytes attenuates diabetic nephropathy. J AM SOC NEPHROL. 2015 2015-05-01;26(5):1115-25.

26. Liu L, Tan J. The relationship between TIMP-1, MMP-9 and diabetic nephropathy. Chin J Clin Rational Drug Use, 2014 7(29):173-174,2014.

27. Said E, Zaitone SA, Eldosoky M, Elsherbiny NM. Nifuroxazide, a STAT3 inhibitor, mitigates inflammatory burden and protects against diabetes-induced nephropathy in rats. Chem Biol Interact. 2018 2018-02-01;281:111-20.

28. Huang W, Gou F, Long Y, Li Y, Feng H, Zhang Q, et al. High Glucose and Lipopolysaccharide Activate NOD1- RICK-NF-kappaB Inflammatory Signaling in Mesangial Cells. Exp Clin Endocrinol Diabetes. 2016 2016-09-01;124(8):512-7.

29. Donate-Correa J, Luis-Rodriguez D, Martin-Nunez E, Tagua VG, Hernandez-Carballo C, Ferri C, et al. Inflammatory Targets in Diabetic Nephropathy. J CLIN MED. 2020 2020-02-07;9(2).

30. Xiao S, Li Q, Hu L, Yu Z, Yang J, Chang Q, et al. Soluble Guanylate Cyclase Stimulators and Activators: Where are We and Where to Go? Mini Rev Med Chem. 2019 2019-01-20;19(18):1544-57.

31. Sakamoto K, Kuno K, Takemoto M, He P, Ishikawa T, Onishi S, et al. Pituitary adenylate cyclase-activating polypeptide protects glomerular podocytes from inflammatory injuries. J DIABETES RES.2015 2015-01-20;2015:727152.

32. Wang G, Yan Y, Xu N, Hui Y, Yin D. Upregulation of microRNA-424 relieved diabetic nephropathy by targeting Rictor through mTOR Complex2/Protein Kinase B signaling. J CELL PHYSIOL. 2019 2019-07-01;234(7):11646-53.

33. Wang RM, Wang ZB, Wang Y, Liu WY, Li Y, Tong LC, et al. Swiprosin-1 Promotes Mitochondria-Dependent Apoptosis of Glomerular Podocytes via P38 MAPK Pathway in Early-Stage Diabetic Nephropathy. CELL PHYSIOL BIOCHEM. 2018 2018-01-20;45(3):899-916.

34. Inada A, Inada $\mathrm{O}$, Fujii $\mathrm{NL}$, Nagafuchi $\mathrm{S}$, Katsuta $\mathrm{H}$, Yasunami $\mathrm{Y}$, et al. Adjusting the 17beta-Estradiol-to-Androgen Ratio Ameliorates Diabetic Nephropathy. J AM SOC NEPHROL. 2016 2016-10-01;27(10):3035-50.

35. Omote K, Gohda T, Murakoshi M, Sasaki Y, Kazuno S, Fujimura T, et al. Role of the TNF pathway in the progression of diabetic nephropathy in KK-A(y) mice. Am J Physiol Renal Physiol. 2014 2014-06-01;306(11):F1335-47.

36. Bohuslavova R, Cerychova R, Nepomucka K, Pavlinkova G. Renal injury is accelerated by global hypoxia-inducible factor 1 alpha deficiency in a mouse model of STZ-induced diabetes. BMC ENDOCR DISORD. 2017 2017-08-03;17(1):48.

37. Wu XY, Yu J, Tian HM. Effect of SOCS1 on diabetic renal injury through regulating TLR signaling pathway. Eur Rev Med Pharmacol Sci. 2019 2019-09-01;23(18):8068-74.

38. Hong YA, Lim JH, Kim MY, Kim Y, Park HS, Kim HW, et al. Extracellular Superoxide Dismutase Attenuates Renal Oxidative Stress Through the Activation of Adenosine Monophosphate-Activated Protein Kinase in Diabetic Nephropathy. Antioxid Redox Signal. 2018 2018-06-10;28(17):1543-61.

39. Yan R, Wang Y, Shi M, Xiao Y, Liu L, Liu L, et al. Regulation of PTEN/AKT/FAK pathways by 
PPARgamma impacts on fibrosis in diabetic nephropathy. J CELL BIOCHEM. 2019 2019-01-16.

40. Luan P, Zhuang J, Zou J, Li H, Shuai P, Xu X, et al. NLRC5 deficiency ameliorates diabetic nephropathy through alleviating inflammation. FASEB J. 2018 2018-02-01;32(2):1070-84.

41. Jiang WY. Therapeutic wisdom in traditional Chinese medicine: a perspective from modern science. TRENDS PHARMACOL SCI. 2005 2005-11-01;26(11):558-63. 


\section{Figures}

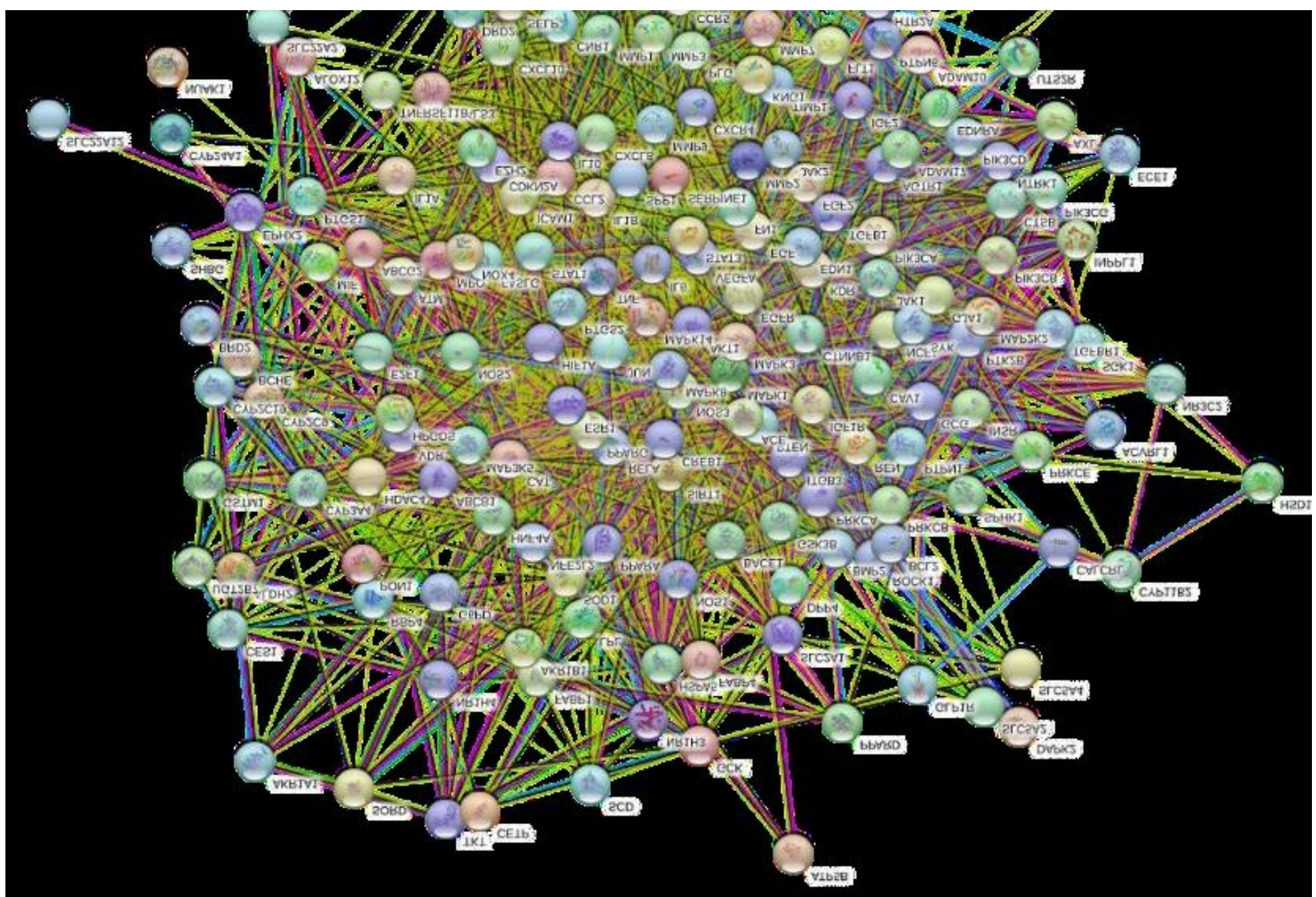

Figure 1

PPI network of the 182 potential targets 


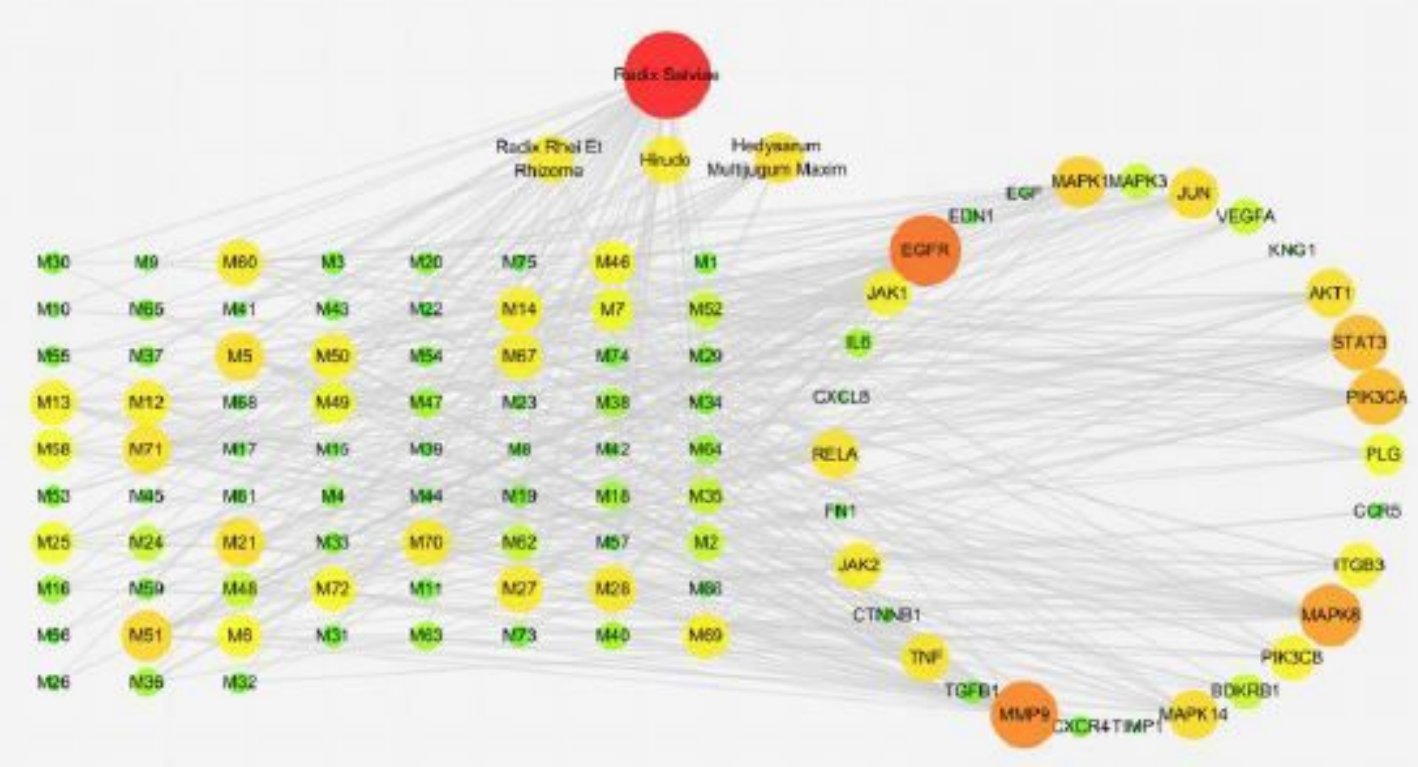

Figure 2

"Drugs - DN - key targets" network. The nodes were visualized with degree. The larger and the redder the node, the higher the degree it was. M1-75 stand for the active ingredients which specific names were shown at appendix B. 


\section{Top 20 of GO Enrichment}

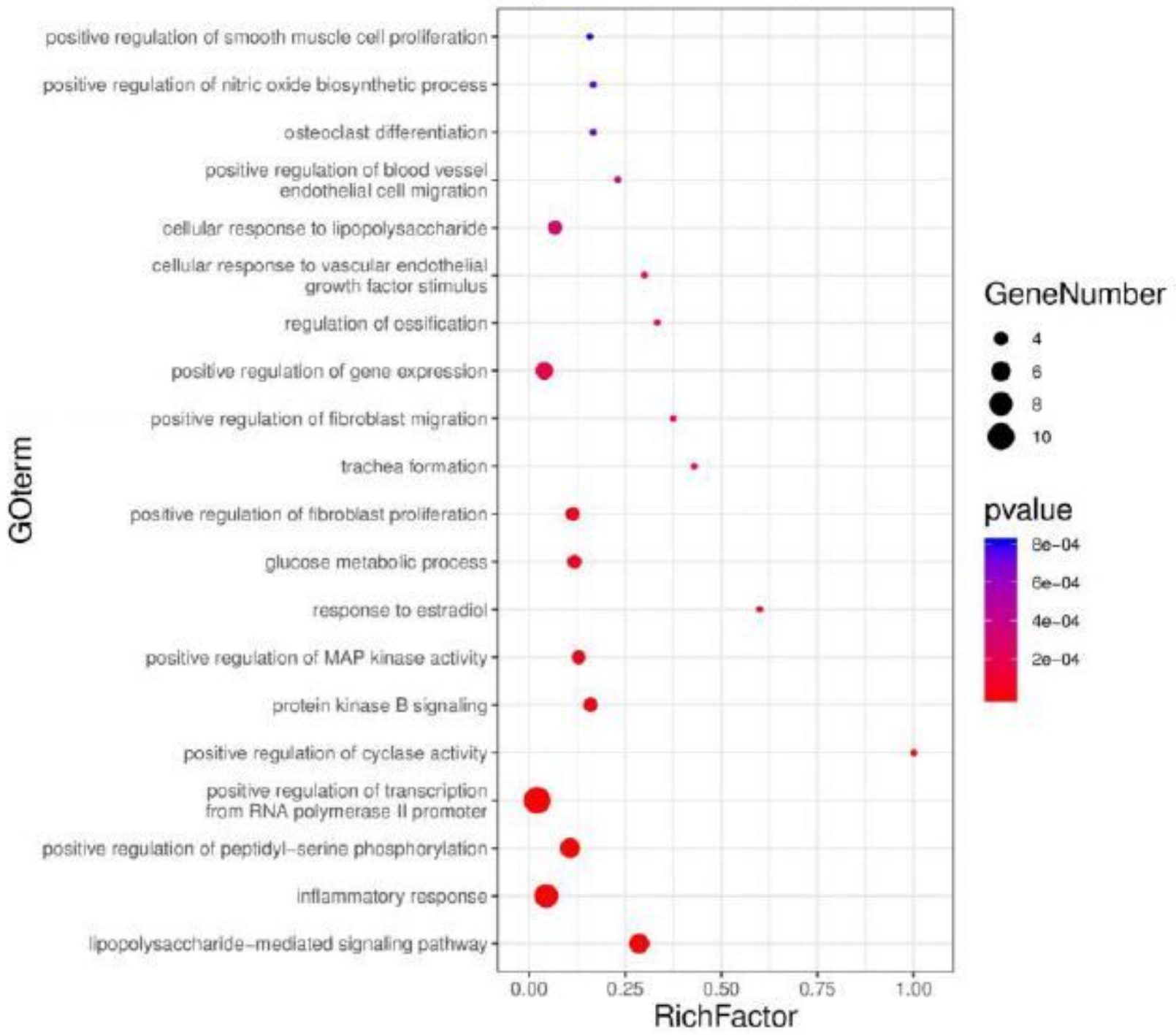

Figure 3

Top 20 enrichments in $\mathrm{GO}$ analysis 


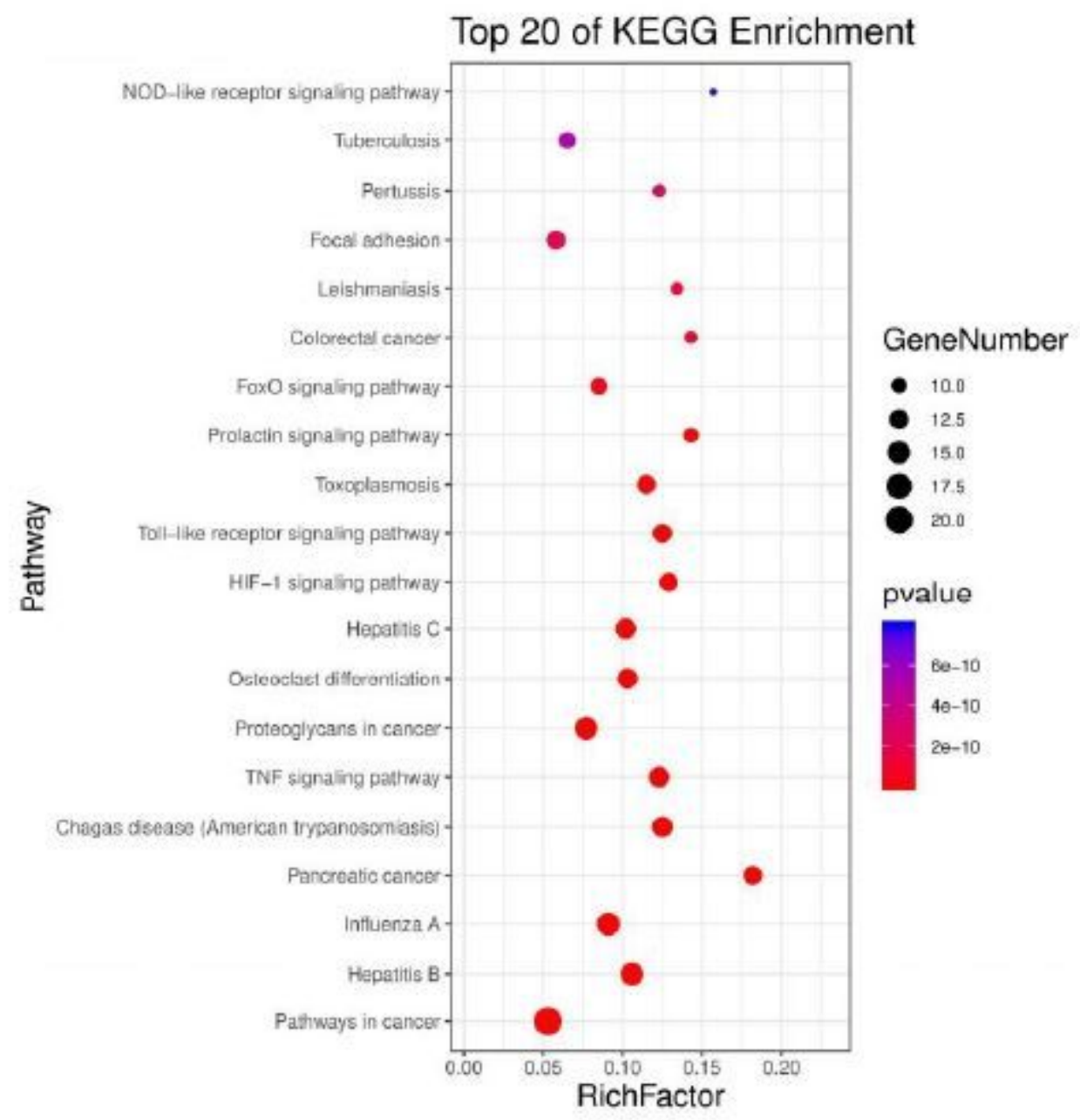

Figure 4

Top 20 KEGG pathway enrichments

\section{Supplementary Files}

This is a list of supplementary files associated with this preprint. Click to download.

- SupplementaryMaterials.xls 